\title{
HAWC results on TeV emitting blazars
}

\author{
Alberto Carramiñana, ${ }^{a, *}$ Daniel Rosa González, ${ }^{a}$ Anna Lia Longinotti, ${ }^{b}$ Fernando \\ Ureña $^{a}$ and Sara Coutiño de León ${ }^{a}$ on behalf of the HAWC Collaboration \\ (a complete list of authors can be found at the end of the proceedings) \\ ${ }^{a}$ Instituto Nacional de Astrofísica, Óptica y Electrónica \\ Luis Enrique Erro 1, Tonantzintla, Puebla, México \\ ${ }^{b}$ Instituto de Astronomía, Universidad Nacional Autónoma de México \\ Ciudad Universitaria, Ciudad de México, México \\ E-mail: alberto@inaoep.mx
}

\begin{abstract}
Since starting full operations, the HAWC $\gamma$-ray observatory has detected and monitored $\mathrm{TeV}$ emission of the nearby BL Lac objects Markarian 421 and Markarian 501. The HAWC Collaboration presented a follow-survey of Active Galactic Nuclei selected from the 3FHL Fermi Catalog covering $60 \%$ of the sky and up to redshift $z \leq 0.3$. Using over 4.5 years of HAWC data we found low-significance evidence for persistent $\mathrm{TeV}$ emission from other sources previously reported as flaring very-high energy sources.
\end{abstract}

\footnotetext{
*** 37th International Cosmic Ray Conference (ICRC2021), ***

*** 12-23 July 2021 ***

*** Berlin, Germany - Online ***
}

\footnotetext{
${ }^{*}$ Presenter
} 


\section{Introduction}

A survey of active galactic nuclei (AGN) performed with the High Altitude Water Cherenkov (HAWC) $\gamma$-ray observatory was presented in [1]. That study was restricted to AGN within the predefined $40^{\circ}$ field of view of HAWC and a redshift $z \leq 0.3$. It confirmed the detections of Markarian 421 and Markarian 501, and gave a maximum-likelihood test-statistic TS $\gtrsim 9$ evidence for TeV $\gamma$-rays from M87, TXS $0518+211$ and TXS 1215+303. These five objects are part of the 32 $\mathrm{GeV} \gamma$-ray active galactic nuclei (AGN) with positive TEV flags in the Third Catalog of Hard Fermi sources (3FHL), indicative of previously reported detections by atmospheric Cherenkov telescopes (ACT) prior to the publication of the 3FHL [2]. The positively flagged TeV sources did not account for the detection of 3C 264, reported shortly after the publication of the 3FHL by [3].

Extreme high-frequency peaked BL Lac objects (EHBL), defined in [4] show a synchrotron peak in the X-ray band, $h v_{\text {sync }}>1 \mathrm{keV}$, and most of them a hard spectral index $(\leq 2)$. Recently, the MAGIC collaboration performed a study of EHBL sources reporting the detection of several AGN flagged as candidates (C) in the 3FHL catalog [5]. In here we revise the HAWC result on known $\mathrm{TeV}$ emitting AGN, accounting for the MAGIC results.

\section{TeV AGN}

This work follows on the survey performed in [1], restricted to HAWC pass- 4 data in the declination range $\delta \in\left[+59^{\circ},-21^{\circ}\right]$ for blazars within a redshift limit $z \leq 0.3$. Table 1 lists the objects studied by MAGIC [5], together with other known EHBL [6] that are within the HAWC sample. Some objects from [5] are not included as they do not conform the HAWC criteria: for example TXS 0637-128 has no known redshift. And RBS 0921 is not a 3FHL source. One important VHE addition from MAGIC is TXS 0210+515, a non-statistical warm-spot in the HAWC data. H 1426+428 appears to have had activity period in 2012 but was not detected by MAGIC. Other blazars are known to have temporary EHBL behavior, like Mrk 501 and 1ES 2344+514, but were not considered here. The sample in table 1 has a mean significance of $\mu[\sqrt{T S}]=+0.53$, which is only $0.15 \sigma$ above null-expectation.

\section{Summary}

None of the EHBL objects studied by MAGIC or from the sample of [6] is detected by HAWC. Furthermore, there is no evidence for emission when taking all the sample together. One aspect to consider is the different energy responses of ACTs and HAWC. We also note that redshift and declination both are important factors for HAWC detectability. Another difference is the duty cycle of EHBL which are known to be flaring sources but may not have long-term persistent emission at a detectable level.

HAWC has revised its low energy response and the data are now been reprocessed (pass A). The study of AGN is one area where an improved sub-TeV sensitivity is due to have a big impact. 


\begin{tabular}{llccc}
\hline \hline 3FHL name & Counterpart & $z$ & TEV flag (VHE) & $T S$ \\
\hline \hline 3FHL J0214.5+5145 & TXS 0210+515 & 0.049 & $\mathrm{C} \mathrm{(Y)}$ & +2.63 \\
3FHL J0232.8+2017 & 1ES 0229+200 & 0.140 & $\mathrm{P}(\mathrm{Y})$ & -0.28 \\
3FHL J0349.3-1159 & 1ES 0347-121 & 0.185 & $\mathrm{P}$ & +0.74 \\
3FHL J0416.8+0105 & 1ES 0414+009 & 0.287 & $\mathrm{P}$ & +1.71 \\
3FHL J0809.7+3457 & BZB J0809+3455 & 0.083 & $\mathrm{C}(\mathrm{N})$ & +0.27 \\
3FHL J0847.2+1134 & RBS 0723 & 0.198 & $\mathrm{P}$ & +0.09 \\
3FHL J0930.4+4952 & 1ES 0927+500 & 0.187 & $\mathrm{~N}(\mathrm{~N})$ & -4.58 \\
3FHL J1221.3+3010 & PG 1218+304 & 0.184 & $\mathrm{P}$ & +5.02 \\
3FHL J1428.5+4240 & H 1426+428 & 0.129 & $\mathrm{P}(\mathrm{N})$ & +1.58 \\
3FHL J2039.4+5219 & 1ES 2037+521 & 0.054 & $\mathrm{P}(\mathrm{Y})$ & -0.94 \\
3FHL J2042.0+2428 & RGB J2042+244 & 0.104 & $\mathrm{C}(\mathrm{Y} ?)$ & +0.58 \\
3FHL J2314.0+1445 & RGB J2313+147 & 0.162 & $\mathrm{C}(\mathrm{N})$ & +1.37 \\
\hline
\end{tabular}

Table 1: EHBL sources in the HAWC AGN survey. Parenthesis letter on $\mathrm{TeV}=\mathrm{C}$ or $\mathrm{N}$ sources indicates detection or non-detection by MAGIC [5].

\section{References}

[1] Albert, A., et al., 2021, ApJ 907, 67.

[2] Ajello, M., et al., 2017, ApJS 232, 18.

[3] Murkherjee, R. for the VERITAS Collaboration, 2018, Astronomers Telegram 11436.

[4] Costamante, L., et al., 2001, A\&A 371, 512.

[5] Acciari, V.A., et al., 2020, ApJS 247, 16.

[6] Costamante, L., et al., 2018, MNRAS 477, 4257.

\section{Acknowledgements}

We acknowledge the support from: the US National Science Foundation (NSF); the US Department of Energy Office of High-Energy Physics; the Laboratory Directed Research and Development (LDRD) program of Los Alamos National Laboratory; Consejo Nacional de Ciencia y Tecnología (CONACyT), México, grants 271051, 232656, 260378, 179588, 254964, 258865, 243290, 132197, A1-S-46288, A1-S-22784, cátedras 873, 1563, 341, 323, Red HAWC, México; DGAPA-UNAM grants IG101320, IN111716-3, IN111419, IA102019, IN110621, IN110521; VIEP-BUAP; PIFI 2012, 2013, PROFOCIE 2014, 2015; the University of Wisconsin Alumni Research Foundation; the Institute of Geophysics, Planetary Physics, and Signatures at Los Alamos National Laboratory; Polish Science Centre grant, DEC-2017/27/B/ST9/02272; Coordinación de la Investigación Científica de la Universidad Michoacana; Royal Society - Newton Advanced Fellowship 180385; Generalitat Valenciana, grant CIDEGENT/2018/034; Chulalongkorn University's CUniverse (CUAASC) grant; Coordinación General Académica e Innovación (CGAI-UdeG), PRODEP-SEP UDG-CA499; Institute of Cosmic Ray Research (ICRR), University of Tokyo, H.F. acknowledges support by NASA under award number 80GSFC21M0002. We also acknowledge the significant contributions over many years of Stefan Westerhoff, Gaurang Yodh and Arnulfo Zepeda Dominguez, all deceased members of the HAWC collaboration. Thanks to Scott Delay, Luciano Díaz and Eduardo Murrieta for technical support. 


\section{Full Authors List: HAWC HAWC}

A.U. Abeysekara ${ }^{48}$, A. Albert ${ }^{21}$, R. Alfaro ${ }^{14}$, C. Alvarez ${ }^{41}$, J.D. Álvarez ${ }^{40}$, J.R. Angeles Camacho ${ }^{14}$, J.C. Arteaga-Velázquez ${ }^{40}$, K. P. Arunbabu $^{17}$, D. Avila Rojas ${ }^{14}$, H.A. Ayala Solares ${ }^{28}$, R. Babu ${ }^{25}$, V. Baghmanyan ${ }^{15}$, A.S. Barber ${ }^{48}$, J. Becerra Gonzalez ${ }^{11}$, E. BelmontMoreno $^{14}$, S.Y. BenZvi ${ }^{29}$, D. Berley ${ }^{39}$, C. Brisbois ${ }^{39}$, K.S. Caballero-Mora ${ }^{41}$, T. Capistrán ${ }^{12}$, A. Carramiñana ${ }^{18}$, S. Casanova ${ }^{15}$, O. Chaparro-Amaro $^{3}$, U. Cotti ${ }^{40}$, J. Cotzomi $^{8}$, S. Coutiño de León ${ }^{18}$, E. De la Fuente ${ }^{46}$, C. de León ${ }^{40}$, L. Diaz-Cruz ${ }^{8}$, R. Diaz Hernandez ${ }^{18}$, J.C. Díaz-Vélez ${ }^{46}$, B.L. Dingus ${ }^{21}$, M. Durocher ${ }^{21}$, M.A. DuVernois ${ }^{45}$, R.W. Ellsworth ${ }^{39}$, K. Engel ${ }^{39}$, C. Espinoza ${ }^{14}$, K.L. Fan ${ }^{39}$, K. Fang $^{45}$, M. Fernández Alonso ${ }^{28}$, B. Fick ${ }^{25}$, H. Fleischhack ${ }^{51,11,52}$, J.L. Flores ${ }^{46}$, N.I. Fraija ${ }^{12}$, D. Garcia ${ }^{4}$, J.A. García-González ${ }^{20}$, J. L. García-Luna ${ }^{46}$, G. García-Torales ${ }^{46}$, F. Garfias ${ }^{12}$, G. Giacinti ${ }^{22}$, H. Goksu ${ }^{22}$, M.M. González ${ }^{12}$, J.A. Goodman ${ }^{39}$, J.P. Harding ${ }^{21}$, S. Hernandez ${ }^{14}$, I. Herzog ${ }^{25}$, J. Hinton ${ }^{22}$, B. Hona ${ }^{48}$, D. Huang ${ }^{25}$, F. Hueyotl-Zahuantitla ${ }^{41}$, C.M. Hui ${ }^{23}$, B. Humensky ${ }^{39}$, P. Hüntemeyer ${ }^{25}$, A. Iriarte ${ }^{12}$, A. Jardin-Blicq ${ }^{22,49,50}$, H. Jhee ${ }^{43}$, V. Joshi ${ }^{7}$, D. Kieda ${ }^{48}$, G J. Kunde ${ }^{21}$, S. Kunwar ${ }^{22}$, A. Lara ${ }^{17}$, J. Lee ${ }^{43}$, W.H. Lee ${ }^{12}$, D. Lennarz ${ }^{9}$, H. León Vargas ${ }^{14}$, J. Linnemann ${ }^{24}$, A.L. Longinotti ${ }^{12}$, R. López-Coto ${ }^{19}$, G. Luis-Raya ${ }^{44}$, J. Lundeen ${ }^{24}$, K. Malone ${ }^{21}$, V. Marandon $^{22}$, O. Martinez ${ }^{8}$, I. Martinez-Castellanos ${ }^{39}$, H. Martínez-Huerta ${ }^{38}$, J. Martínez-Castro ${ }^{3}$, J.A.J. Matthews ${ }^{42}$, J. McEnery ${ }^{11}$, P. Miranda-Romagnoli $^{34}$, J.A. Morales-Soto ${ }^{40}$, E. Moreno ${ }^{8}$, M. Mostafá ${ }^{28}$, A. Nayerhoda ${ }^{15}$, L. Nellen ${ }^{13}$, M. Newbold ${ }^{48}$, M.U. Nisa ${ }^{24}$, R. Noriega-Papaqui ${ }^{34}$, L. Olivera-Nieto ${ }^{22}$, N. Omodei ${ }^{32}$, A. Peisker ${ }^{24}$, Y. Pérez Araujo ${ }^{12}$, E.G. Pérez-Pérez ${ }^{44}$, C.D. Rho ${ }^{43}$, C. Rivière ${ }^{39}$, D. Rosa-Gonzalez $^{18}$, E. Ruiz-Velasco ${ }^{22}$, J. Ryan ${ }^{26}$, H. Salazar ${ }^{8}$, F. Salesa Greus ${ }^{15,53}$, A. Sandoval ${ }^{14}$, M. Schneider ${ }^{39}$, H. Schoorlemmer ${ }^{22}$, J. Serna-Franco ${ }^{14}$, G. Sinnis ${ }^{21}$, A.J. Smith ${ }^{39}$, R.W. Springer ${ }^{48}$, P. Surajbali ${ }^{22}$, I. Taboada ${ }^{9}$, M. Tanner ${ }^{28}$, K. Tollefson ${ }^{24}$, I. Torres ${ }^{18}$, R. Torres-Escobedo $^{30}$, R. Turner ${ }^{25}$, F. Ureña-Mena ${ }^{18}$, L. Villaseñor ${ }^{8}$, X. Wang ${ }^{25}$, I.J. Watson ${ }^{43}$, T. Weisgarber ${ }^{45}$, F. Werner ${ }^{22}$, E. Willox ${ }^{39}$, J. Wood ${ }^{23}$, G.B. Yodh ${ }^{35}$, A. Zepeda ${ }^{4}$, H. Zhou ${ }^{30}$

${ }^{1}$ Barnard College, New York, NY, USA, ${ }^{2}$ Department of Chemistry and Physics, California University of Pennsylvania, California, PA, USA, ${ }^{3}$ Centro de Investigación en Computación, Instituto Politécnico Nacional, Ciudad de México, México, ${ }^{4}$ Physics Department, Centro de Investigación y de Estudios Avanzados del IPN, Ciudad de México, México, ${ }^{5}$ Colorado State University, Physics Dept., Fort Collins, CO, USA, ${ }^{6}$ DCI-UDG, Leon, Gto, México, ${ }^{7}$ Erlangen Centre for Astroparticle Physics, Friedrich Alexander Universität, Erlangen, BY, Germany, ${ }^{8}$ Facultad de Ciencias Físico Matemáticas, Benemérita Universidad Autónoma de Puebla, Puebla, México, ${ }^{9}$ School of Physics and Center for Relativistic Astrophysics, Georgia Institute of Technology, Atlanta, GA, USA, ${ }^{10}$ School of Physics Astronomy and Computational Sciences, George Mason University, Fairfax, VA, USA, ${ }^{11}$ NASA Goddard Space Flight Center, Greenbelt, MD, USA, ${ }^{12}$ Instituto de Astronomía, Universidad Nacional Autónoma de México, Ciudad de México, México, ${ }^{13}$ Instituto de Ciencias Nucleares, Universidad Nacional Autónoma de México, Ciudad de México, México, ${ }^{14}$ Instituto de Física, Universidad Nacional Autónoma de México, Ciudad de México, México, ${ }^{15}$ Institute of Nuclear Physics, Polish Academy of Sciences, Krakow, Poland, ${ }^{16}$ Instituto de Física de São Carlos, Universidade de São Paulo, São Carlos, SP, Brasil, ${ }^{17}$ Instituto de Geofísica, Universidad Nacional Autónoma de México, Ciudad de México, México, ${ }^{18}$ Instituto Nacional de Astrofísica, Óptica y Electrónica, Tonantzintla, Puebla, México, ${ }^{19}$ INFN Padova, Padova, Italy, ${ }^{20}$ Tecnologico de Monterrey, Escuela de Ingeniería y Ciencias, Ave. Eugenio Garza Sada 2501, Monterrey, N.L., 64849, México, ${ }^{21}$ Physics Division, Los Alamos National Laboratory, Los Alamos, NM, USA, ${ }^{22}$ Max-Planck Institute for Nuclear Physics, Heidelberg, Germany, ${ }^{23}$ NASA Marshall Space Flight Center, Astrophysics Office, Huntsville, AL, USA, ${ }^{24}$ Department of Physics and Astronomy, Michigan State University, East Lansing, MI, USA, ${ }^{25}$ Department of Physics, Michigan Technological University, Houghton, MI, USA, ${ }^{26}$ Space Science Center, University of New Hampshire, Durham, NH, USA, ${ }^{27}$ The Ohio State University at Lima, Lima, OH, USA, ${ }^{28}$ Department of Physics, Pennsylvania State University, University Park, PA, USA, ${ }^{29}$ Department of Physics and Astronomy, University of Rochester, Rochester, NY, USA, ${ }^{30}$ Tsung-Dao Lee Institute and School of Physics and Astronomy, Shanghai Jiao Tong University, Shanghai, China, ${ }^{31}$ Sungkyunkwan University, Gyeonggi, Rep. of Korea, ${ }^{32}$ Stanford University, Stanford, CA, USA, ${ }^{33}$ Department of Physics and Astronomy, University of Alabama, Tuscaloosa, AL, USA, ${ }^{34}$ Universidad Autónoma del Estado de Hidalgo, Pachuca, Hgo., México, ${ }^{35}$ Department of Physics and Astronomy, University of California, Irvine, Irvine, CA, USA, ${ }^{36}$ Santa Cruz Institute for Particle Physics, University of California, Santa Cruz, Santa Cruz, CA, USA, ${ }^{37}$ Universidad de Costa Rica, San José, Costa Rica, ${ }^{38}$ Department of Physics and Mathematics, Universidad de Monterrey, San Pedro Garza García, N.L., México, ${ }^{39}$ Department of Physics, University of Maryland, College Park, MD, USA, ${ }^{40}$ Instituto de Física y Matemáticas, Universidad Michoacana de San Nicolás de Hidalgo, Morelia, Michoacán, México, ${ }^{41}$ FCFM-MCTP, Universidad Autónoma de Chiapas, Tuxtla Gutiérrez, Chiapas, México, ${ }^{42}$ Department of Physics and Astronomy, University of New Mexico, Albuquerque, NM, USA, ${ }^{43}$ University of Seoul, Seoul, Rep. of Korea, ${ }^{44}$ Universidad Politécnica de Pachuca, Pachuca, Hgo, México, ${ }^{45}$ Department of Physics, University of Wisconsin-Madison, Madison, WI, USA, ${ }^{46}$ CUCEI, CUCEA, Universidad de Guadalajara, Guadalajara, Jalisco, México, ${ }^{47}$ Universität Würzburg, Institute for Theoretical Physics and Astrophysics, Würzburg, Germany, ${ }^{48}$ Department of Physics and Astronomy, University of Utah, Salt Lake City, UT, USA, ${ }^{49}$ Department of Physics, Faculty of Science, Chulalongkorn University, Pathumwan, Bangkok 10330, Thailand, ${ }^{50}$ National Astronomical Research Institute of Thailand (Public Organization), Don Kaeo, MaeRim, Chiang Mai 50180, Thailand, ${ }^{51}$ Department of Physics, Catholic University of America, Washington, DC, USA, ${ }^{52}$ Center for Research and Exploration in Space Science and Technology, NASA/GSFC, Greenbelt, MD, USA, ${ }^{53}$ Instituto de Física Corpuscular, CSIC, Universitat de València, Paterna, Valencia, Spain 